Case Report

\title{
Two Surgical Cases of Combined Hepatocellular-Cholangiocarcinoma, Intermediate-Cell Subtype: Potentially Characteristic Gross Features
}

\author{
Jiro Watanabe, ${ }^{1}$ Sohsuke Yamada $\mathbb{D}^{1},{ }^{1,2}$ Yasuyuki Sasaguri, ${ }^{1,3}$ \\ Masaru Inagaki, ${ }^{4}$ and Hiromi Iwagaki ${ }^{4}$ \\ ${ }^{1}$ Laboratory of Pathology, National Hospital Organization, Fukuyama Medical Center, Fukuyama, Japan \\ ${ }^{2}$ Department of Pathology and Laboratory Medicine, Kanazawa Medical University, 1-1 Uchinada, Ishikawa 920-0293, Japan \\ ${ }^{3}$ Laboratory of Pathology, Fukuoka Tokushukai Hospital, Fukuoka, Japan \\ ${ }^{4}$ Department of Surgery, National Hospital Organization, Fukuyama Medical Center, Fukuyama, Japan \\ Correspondence should be addressed to Sohsuke Yamada; sohsuke@kanazawa-med.ac.jp
}

Received 2 February 2018; Accepted 12 April 2018; Published 5 June 2018

Academic Editor: Tibor Tot

Copyright (C) 2018 Jiro Watanabe et al. This is an open access article distributed under the Creative Commons Attribution License, which permits unrestricted use, distribution, and reproduction in any medium, provided the original work is properly cited.

\begin{abstract}
We herein reported two rare surgical cases of primary combined hepatocellular-cholangiocellular carcinoma, intermediatecell subtype (CHC-INT), showing potentially characteristic and specific gross findings on their cut surface: both CHC-INTs demonstrated poorly demarcated and expansive and/or infiltrative hepatic nodules in lobulated margins, appearing clearly whitish in color. We were finally able to accurately diagnose the current lesions after thorough analyses including an appropriate and wide panel of immunohistochemical antibodies. Despite that, all pathologists should be aware that the potentially characteristic gross features of primary CHC-INT might also be one of the powerful supplementary tools for reaching its correct, conclusive diagnosis.
\end{abstract}

\section{Introduction}

In 1903, Wells first reported the features of primary malignant liver tumor, combined hepatocellular- (HCC-) cholangiocellular (CCC) carcinoma (CHC), and following that, Allen and Lisa, Goodman et al., and Taguchi et al. subclassified CHC variously [1-3]. Recently, in the latest World Health Organization (WHO) classification of the digestive system, $\mathrm{CHC}$ is ultimately divided into (1) classical type and (2) subtypes with stem cell features [4]. Furthermore, the latter (2) has been subdivided into (i) typical subtype, (ii) intermediate-cell subtype (CHC-INT), and (iii) cholangiolocellular subtype [4]. In particular, the histopathological findings of rare (ii) CHC-INT characteristically demonstrate a proliferation of relatively small and uniform carcinoma cells having intermediate features between hepatocytes and cholangiocytes, i.e., HCC and CCC, arranged in strands, solid nests, trabeculae, and/or ill-defined gland-/tubule-like structures [4]. A substantial number of interesting papers focusing especially on the histopathological and immunohistochemical features of CHC-INT were published [3, 5]; however, within our thorough investigation, there has been no detailed description regarding the gross findings reported in the English literature. Indeed, the above WHO classification has merely stated that the gross morphology of CHC-INT is not significantly different from that of HCC [4]; however, we cannot completely agree with that description. We herein briefly report two rare surgical cases of primary CHC-INT, showing potentially characteristic and specific gross features on their cut surface.

\section{Case Presentation (Case 1)}

The first patient, who was a man in his late seventies with an unremarkable previous medical history, presented with isovascular nodule accompanied by slow venous wash-out on abdominal dynamic CT in the left lobe of liver. The laboratory data, including the blood cell count, chemistry, and tumor 


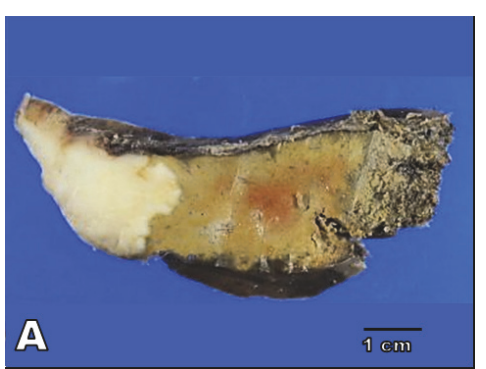

(a)

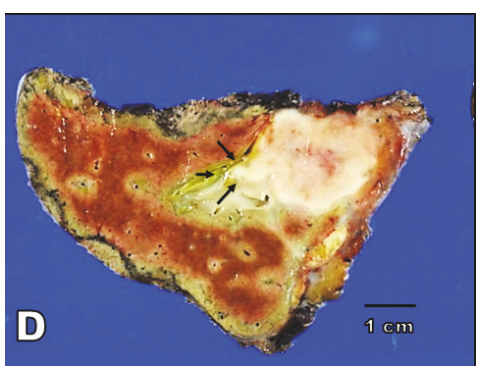

(d)

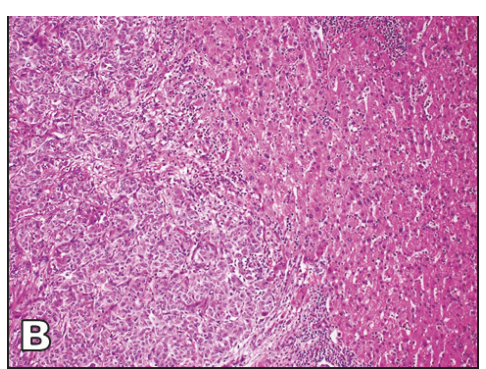

(b)

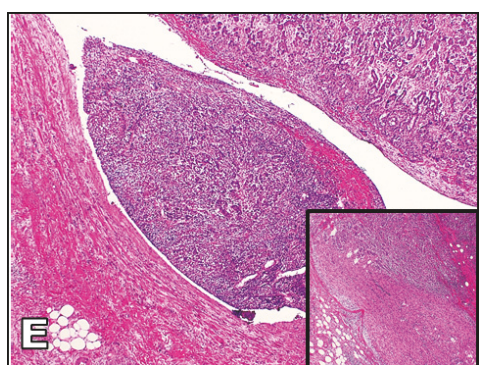

(e)

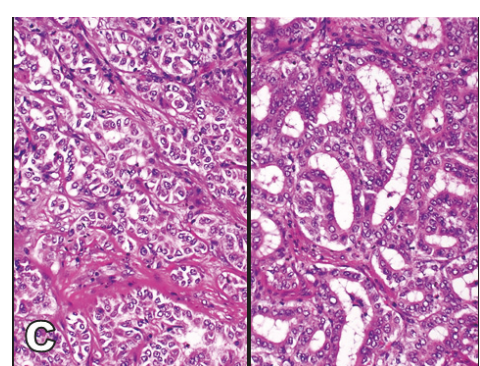

(c)

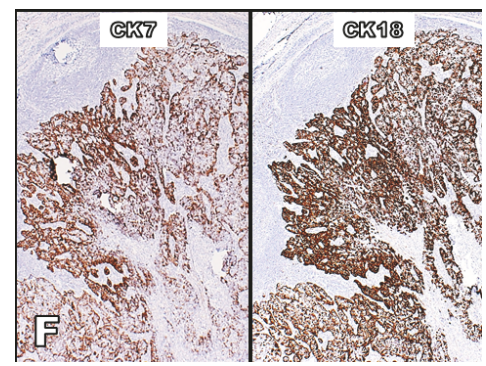

(f)

FIgURE 1: The gross, microscopic, and immunohistochemical examinations of the primary CHC-INT. (a) The cut surface of the first CHC-INT case characteristically shows a poorly demarcated peripheral nodule in lobulated margins, measuring $32 \mathrm{x} 21 \mathrm{~mm}$ in diameter, which appears clearly whitish in color. Bar $=1 \mathrm{~cm}$. (b) A microscopic examination of the first CHC-INT case (H\&E staining) demonstrates an unencapsulated, ill-defined, and expansive nodule (left side), arranged predominantly in a proliferation of solid nests pattern (left side). (c) The first CHC-INT (H\&E staining) shows a solid proliferation of atypical epithelial cells, forming trabeculae (left panel) and/or ill-defined, fused tubule-like structures (right panel). These atypical cells are small-to-medium-sized and relatively uniform, having enlarged hyperchromatic nuclei and scant cytoplasm. (d) The cut surface of the second CHC-INT case characteristically reveals a poorly demarcated portal nodule in lobulated margins with central necrosis, measuring $35 \times 26 \mathrm{~mm}$ in diameter, which appears clearly whitish in color, accompanied by portal vein permeation (arrows). Bar $=1 \mathrm{~cm}$. (e) A microscopic examination of the second unencapsulated CHC-INT tumor (H\&E staining) shows a solid proliferation of atypical epithelial cells, arranged predominantly in solid nests, trabeculae, and/or irregular and fused tubule-like structures, aggressively involving the portal vein (right upper side to center) with focal perineural invasion (inset). Left lower side is the intact wall of the portal vein. (f) Immunohistochemistry demonstrates that those second CHC-INT carcinoma cells are specifically positive for not only CK7 (left; cholangiocytes marker) but also CK18 (marker for both hepatocytes and cholangiocytes).

marker levels, were within the normal limits, with the exception of mildly elevated CRP $(0.39 \mathrm{mg} / \mathrm{dL})$ and decreased hemoglobin $(8.9 \mathrm{~g} / \mathrm{dL})$ levels. Neither infection of HBV nor infection of HCV was noted. Based on the clinical findings, the initial diagnosis by the clinicians was most likely HCC, and, thus, left partial hepatectomy was performed. On gross examination, the cut surface of hepatic nodule (Figure 1(a)) showed a poorly demarcated peripheral nodule in lobulated margins, measuring $32 \times 21 \mathrm{~mm}$ in diameter, which appeared clearly whitish in color. The background of this liver showed no remarkable change (Figure 1(a)). A microscopic examination of the tumor demonstrated an unencapsulated, illdefined, and expansive nodule (Figure 1(b)). This cancerous nodule showed a solid proliferation of atypical epithelial cells, arranged predominantly in solid nests (Figure 1(b)), trabeculae, and/or ill-defined, fused tubule-like structures (Figure 1(c)). On a high-power view, these atypical cells were small-to-medium-sized and relatively uniform, having enlarged hyperchromatic nuclei and scant cytoplasm without any evidence of intracytoplasmic mucin (Figure 1(c)). Intriguingly, prominent fibrous stroma was not evident in this tumor. Immunohistochemistry revealed that the abovementioned carcinoma cells were specifically positive for not only CK7 (cholangiocytes marker) but also CK8/CK18 (markers for both hepatocytes and cholangiocytes) and CK19/CD56 (potential stem cells markers), whereas they were negative for Hepatocyte (hepatocytes marker) $[3,5]$. Based on all of these features, the final diagnosis was primary CHC-INT. To date, this patient has been followed up for 1 year since surgery, and he remains well without any sign of recurrence.

\section{Case Presentation (Case 2)}

The second patient, who was also a man in his early seventies with more than 25-year follow-up for HCV-positive chronic hepatitis and recurrent HCC, presented with mildly hypervascular and ring-enhanced nodule accompanied by venous wash-out on abdominal dynamic CT in the S5 of the remnant liver. The laboratory data were mostly within the normal limits, with the exception of mildly elevated AST (37 IU/L), total bilirubin $(1.8 \mathrm{mg} / \mathrm{dL})$, and CEA $(5.55 \mathrm{ng} / \mathrm{mL})$ levels. No infection of HBV was observed. Based on the clinical 
findings, the initial diagnosis by the clinicians was recurrent HCC, and, thus, partial S5 hepatectomy was performed. On gross examination, the cut surface of hepatic nodule (Figure 1(d)) showed a poorly demarcated portal nodule in lobulated margins with central necrosis, measuring $35 \mathrm{x}$ $26 \mathrm{~mm}$ in diameter, which appeared clearly whitish in color, accompanied by not only gross but also histopathological portal vein permeation (Figure 1(e)). A microscopic examination of the tumor showed an unencapsulated, ill-defined, and expansive/infiltrative nodule, displaying a solid proliferation of atypical epithelial cells, arranged predominantly in solid nests, trabeculae, and/or irregular and fused tubule-like structures, aggressively involving the portal vein with focal perineural invasion (Figure 1(e)). On a high-power view, these atypical cells were very similar to those of the abovementioned first case. Prominent fibrous stroma was not seen either. The background of this liver showed mild chronic hepatitis (F1/A1) and steatosis. Immunohistochemistry showed that those carcinoma cells were specifically positive for not only CK7 (Figure 1(f)) but also CK18 (Figure 1(f)) and ckit (potential stem cells marker) [3,5], whereas they were negative for CK8, Hepatocyte, and CD56. Based on all of these features, the final diagnosis was primary CHC-INT as well. The recurrence of CHC-INT in the remnant liver occurred 1 year and 3 months after this surgery, but he remains not worse with follow-up for the postoperative 2 years.

\section{Discussion}

It is very likely that the current report of two surgical CHCINT patients is clinicopathologically remarkable for one reason at least. In case of the present gross findings for poorly demarcated, unencapsulated, and lobulated nodules looking clearly whitish in color on the cut surface, we pathologists should consider the rare possibility of CHC-INT, rather than common HCC. In addition, a wide panel of immunohistochemical analyses should be critically performed, as shown here. In our opinion, the macroscopic features of HCCs appear yellow-whitish, tan to green, but not clearly whitish, in color, even though further modified by varying degrees of necrosis/hemorrhage and the production of bile or fat. In fact, the most critical differential diagnosis in the present cases was HCC, despite the fact that it should be relatively easy to rule out this possibility through a clinicopathologic examination or immunohistochemistry. Since most CHCs are known to generally have a worse outcome, growing rapidly and showing aggressive/infiltrative behaviours $[3,4,6]$, as in our second case, alerting the surgeons to the postoperative careful follow-up and additional treatment, at the very least, should be raised. Furthermore, Sasaki et al. have recently reported that, among the subtypes with stem cell features of $\mathrm{CHC}$, CHC-INT is significantly associated with bigger tumor size or higher histological grade of coexistent HCC [7]; however, its prognosis after curative surgical resection is unknown, with conflicting evidence based on small series and patients' numbers [3, 4, 6, 7]. Nevertheless, it would be intriguing to assess the significance of those unique gross features and prognoses on future larger studies. This short case report, taken together with the potentially specific findings of cut surface for CHC-INT, might promote interest within the scientific community.

\section{Conclusion}

In conclusion, we herein reported two rare surgical cases of primary CHC-INT, showing potentially characteristic and specific gross findings on their cut surface: both $\mathrm{CHC}$ INTs demonstrated poorly demarcated and expansive and/or infiltrative hepatic nodules in lobulated margins, appearing clearly whitish in color. We were finally able to accurately diagnose the current lesions after thorough analyses including an appropriate and wide panel of immunohistochemical antibodies. Despite that, all pathologists should be aware that the potentially characteristic gross features of primary $\mathrm{CHC}$ INT might also be one of the powerful supplementary tools for reaching its correct, conclusive diagnosis.

\section{Abbreviations \\ CT: $\quad$ Computed tomography \\ HBV: $\quad$ Hepatitis type B virus \\ HCV: Hepatitis type $C$ virus \\ CK: $\quad$ Cytokeratin \\ Hepatocyte: Staining for hepatocyte antigen \\ AST: $\quad$ Aspartate aminotransferase \\ CEA: Carcinoembryonic antigen \\ F1/A1: $\quad$ Fibrosis staging 1/activity grading 1.}

\section{Data Availability}

The dataset supporting the findings and conclusions of this report is included within the article.

\section{Consent}

Written informed consent was obtained from the patients and their families on admission for the publication of this report and any accompanying images.

\section{Conflicts of Interest}

The authors declare no conflicts of interest in association with this study.

\section{Acknowledgments}

This work received fund from the National Hospital Organization, Fukuyama Medical Center, Fukuyama, Japan. The authors would like to thank Dr. and Associate Professor Jun Akiba, Department of Pathology, Kurume University School of Medicine, Kurume, Japan, for his constructive and helpful suggestions/comments and correct diagnoses. 


\section{References}

[1] R. A. Allen and J. R Lisa, "Combined liver cell and bile duct carcinoma," American Journal of Pathology, vol. 25, pp. 647-655, 1949.

[2] J. Taguchi, O. Nakashima, M. Tanaka, T. Hisaka, T. Takazawa, and M. Kojiro, "A Clinicopathological study on combined hepatocellular and cholangiocarcinoma," Journal of Gastroenterology and Hepatology, vol. 11, no. 8, pp. 758-764, 1996.

[3] J. Akiba, O. Nakashima, S. Hattori et al., "Clinicopathologic analysis of combined hepatocellular-cholangiocarcinoma according to the latest who classification," The American Journal of Surgical Pathology, vol. 37, no. 4, pp. 496-505, 2013.

[4] N. D. Theise, O. Nakashima, Y. N. Park, and Y. Nakanuma, WHO Classification of Tumours of the Digestive System, Lyon, France, IARC Press, 2010.

[5] J. Akiba, O. Nakashima, S. Hattori et al., "The expression of arginase-1, keratin (K) 8 and K18 in combined hepatocellularcholangiocarcinoma, subtypes with stem-cell features, intermediate-cell type," Journal of Clinical Pathology, vol. 69, no. 10, pp. 846-851, 2016.

[6] M. Sasaki, Y. Sato, and Y. Nakanuma, "Mutational landscape of combined hepatocellular carcinoma and cholangiocarcinoma, and its clinicopathological significance," Histopathology, vol. 70, no. 3, pp. 423-434, 2017.

[7] M. Sasaki, H. Sato, Y. Kakuda, Y. Sato, J. H. Choi, and Y. Nakanuma, "Clinicopathological significance of 'subtypes with stem-cell feature' in combined hepatocellular-cholangiocarcinoma," Liver International, vol. 35, no. 3, pp. 1024-1035, 2015. 


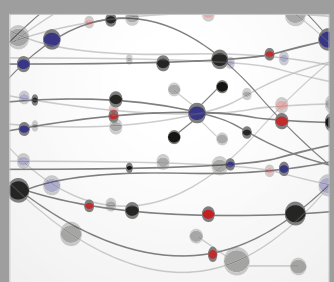

The Scientific World Journal
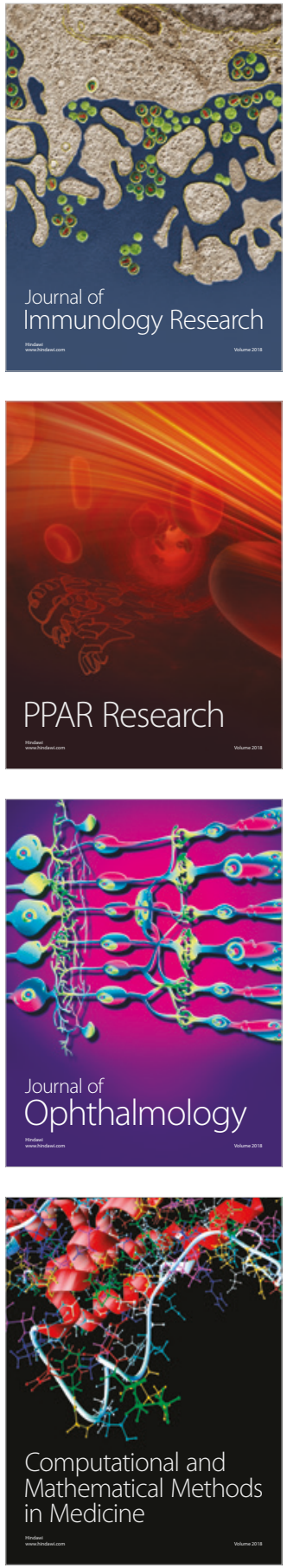

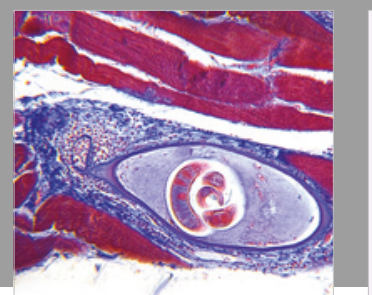

Gastroenterology Research and Practice

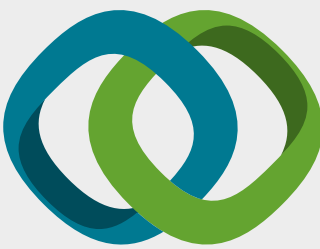

\section{Hindawi}

Submit your manuscripts at

www.hindawi.com
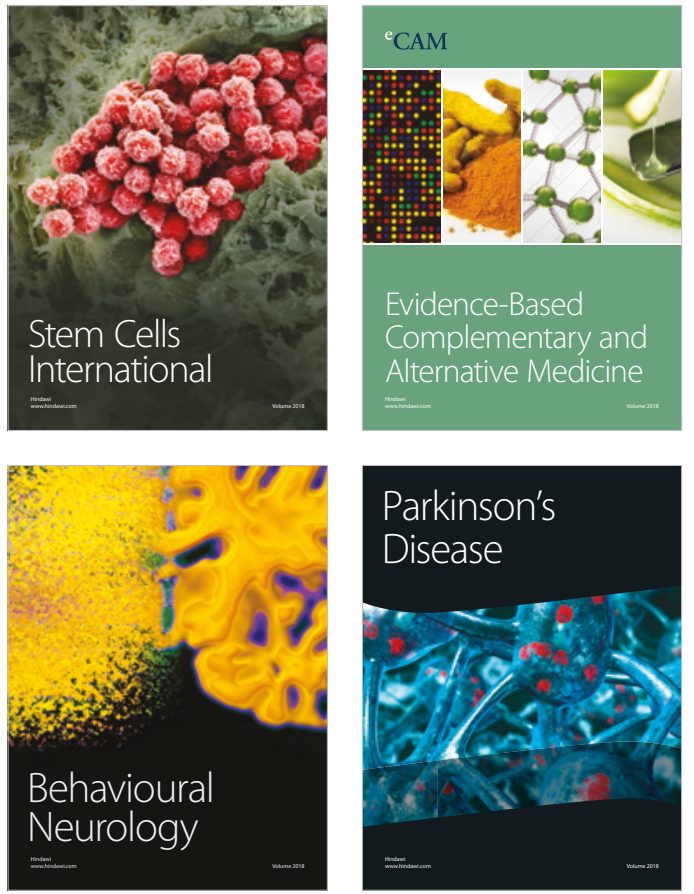

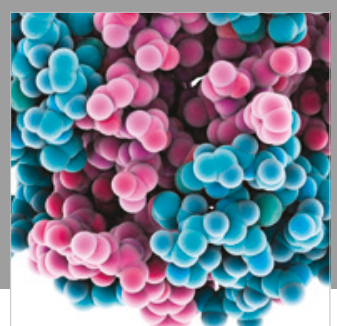

ournal of

Diabetes Research

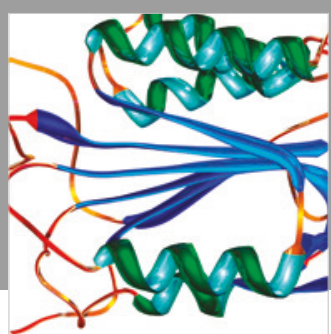

Disease Markers
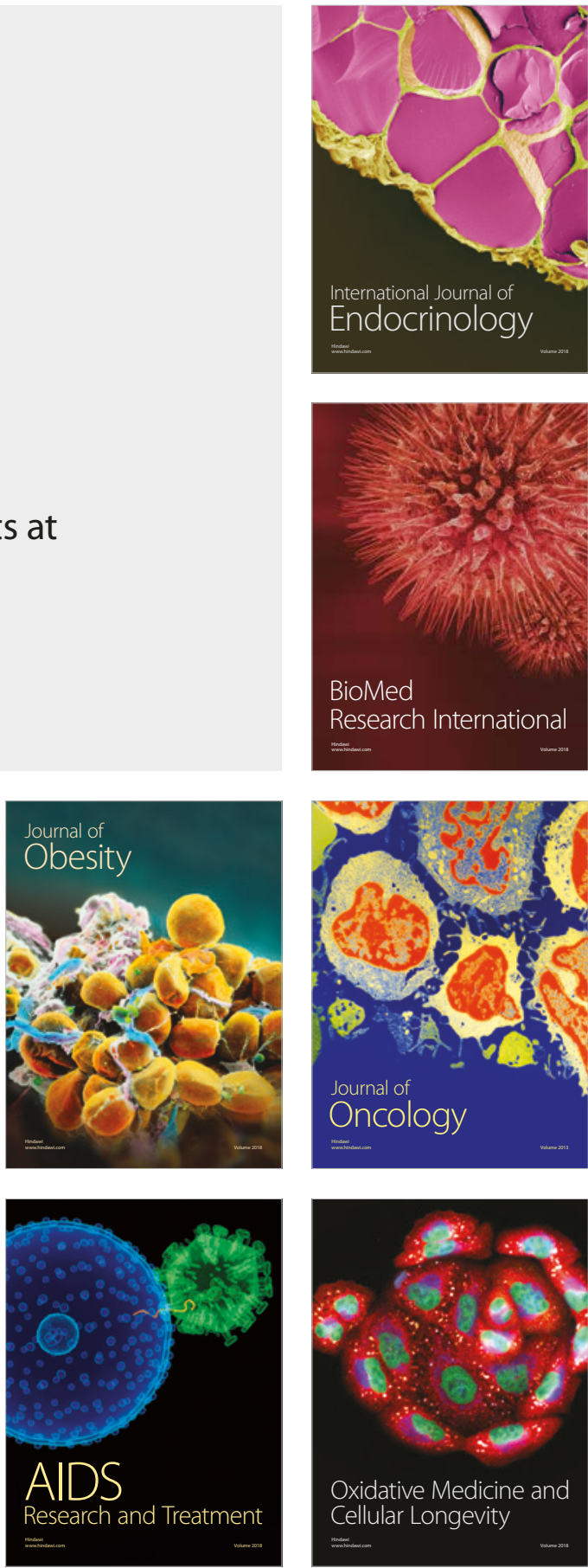